\title{
Infections in IBD patients on immunomodulators, corticosteroids, and anti-TNF therapy: Is elderly age a predictor?
}

\author{
Christina Tofani ${ }^{1 *}$, Faten Aberra ${ }^{2}$, Ann Tierney ${ }^{3}$ and Lichtenstein GR ${ }^{4}$ \\ ${ }^{1}$ Department of Internal Medicine, Hospital University of Pennsylvania, USA \\ ${ }^{2}$ Division of Gastroenterology, Department of Internal Medicine, Hospital of the University of Pennsylvania, USA \\ ${ }^{3}$ Biostatistics Analysis Center, University of Pennsylvania, USA \\ ${ }^{4}$ Division of Gastroenterology, Department of Internal Medicine, Hospital of the University of Pennsylvania, USA
}

\begin{abstract}
Background: Inflammatory bowel disease (IBD) therapy, including immunomodulatory (IMM), corticosteroids (CS), and TNF inhibitors (TNFI), is known to increase the infectious risk when used in to treat many diseases. No recent studies have analyzed the incidence of infections in elderly patients on these therapies. We aim to analyze the effect of immunosuppressant use and age on infectious complications in IBD patients.
\end{abstract}

Methods: A retrospective cohort study was conducted of patients at a tertiary care center with Crohns disease (CD), ulcerative colitis (UC), or IBD, comparing infection risk in patients $\geq 65$ years old to those $<65$ years old. Chi-square and Wilcoxon Rank Sum statistics were performed. Infection rate ratio (IRRs) were evaluated.

Results: 292 patients were assessed. $83 \%$ of patients < 65 years old and $72 \%$ of patients $\geq 65$ years old were on immunosuppressant's. Infections occurred in $41.4 \%$ patients. $7.8 \%$ of the cohort had $\geq 2$ infections. The infection incidence rate per year of follow up in the younger and older cohorts were 0.106 and 0.170 , respectively, with IRR of $1.60(95 \% \mathrm{CI}=1.16,2.21)$ in the older. The IRR in elderly patients on CS compared to non-CS regimens was $3.28(95 \% \mathrm{CI}=1.62,6.64)$. The IRR in elderly patients on IMM and CS, and on TNFI and CS were 10.58 (95\% CI=1.27, 87.91) and 10.64 (95\% CI=1.95, 58.10), respectively.

Conclusions: Increasing age is associated with an increased risk for infections in IBD patients using immunosuppressive medications. CS had the highest IRR for infections compared to other therapies. Infectious risk is greatly increased when CS are added to IMM and TNFI.

\section{Background}

Most patients are diagnosed with inflammatory bowel disease at a young age. Advancements in medicine are affording patients longer lives. Therefore, patients are being diagnosed later in life and more elderly individuals are living with IBD. The 2009 US census estimated that over 1.1 million Americans had IBD, mostly ulcerative colitis or Crohn's disease [1]. About 13\% of patients with IBD are over age 60 [2]. Therapeutic options for IBD include aminosalicylates, antibiotics, and immunosuppressants, such as corticosteroids, immunomodulators (azathioprine, mercaptopurine and methotrexate), TNF inhibitors (infliximab, adalimumab, certolizumab pegol and golimumab), and small adhesion molecules (natalizumab). Elderly patients with inflammatory bowel disease differ from younger patients in some ways that must be considered when diagnosing and treating this population. They have more medical comorbidities and altered pharmacokinetics [3].

IBD therapies have a number of potential side effects. Long term use of corticosteroids has been associated with the development of diabetes mellitus, Cushing's syndrome, and osteoporosis [4]. Side effects of immunomodulators include hepatotoxicity, bone marrow suppression, lymphoma and pancreatitis [5]. TNF inhibitors are associated with increased risk of infections and, rarely, malignancy [6]. Corticosteroids, immunomodulators, and TNF inhibitors alter the immune system and may increase the risk of infectious complications [7-9].

The TREAT registry, established in 2005 as a prospective review of outcomes of patients with Crohn's disease, analyzed independent risk factors for infectious complications in this patient population. Prednisone, narcotics, moderate to severe disease activity and infliximab were considered to be independent risk factors for infection $[10,11]$. Corticosteroids have also been suggested to increase the risk of postoperative infections in IBD patients [12]. In addition, elderly patients on TNF inhibitors are at increased risk for infectious complications when compared to elderly patient not on TNF inhibitors and younger patients that were on TNF inhibitors [13].

More elderly patients are on IBD therapies. Therefore, it is important to critically analyze the safety of these medications in this patient population. The effects of these medications in the

Correspondence to: Christina Tofani, MD, 132 S. 10th St., Main Building, Suite 480, Philadelphia, PA, USA, Tel: +1 215-662-4000; E-mail: christina.tofani@gmail.com

Key words: infections, age, Crohn's, ulcerative, colitis

Received: August 19, 2016; Accepted: September 20, 2016; Published: September 23, 2016 
elderly population are poorly characterized. In elderly IBD patients, corticosteroids increase the risk for surgery in patients with ulcerative colitis [14]. There have been a few small case series in the literature suggesting elderly age may be an independent risk factor for infectious complications in patients with IBD. However, no study has taken corticosteroids, immunomodulators and TNF inhibitors into account and individually analyzed their contribution to this risk. The aim of this study is to analyze whether being elderly places patients with IBD at increased risk of infectious complications when using conventional medical therapy for IBD.

\section{Patients and methods}

Approval was obtained from the Institutional Review Board at the University of Pennsylvania. A retrospective analysis of our electronic medical record was performed of all patients seen in the IBD center at the University of Pennsylvania Health System. We included patients aged 18 years and older who carried a diagnosis of Crohn's disease (CD), ulcerative colitis (UC), or indeterminate colitis (IC) and had been evaluated at the University of Pennsylvania Health System between January 1, 2007 and November 1, 2012. The following data were assessed: age, sex, diagnosis, treatment regimen (mesalamine, antibiotics, corticosteroids, immunomodulators [6-MP, Azathioprine or methotrexate] or Anti-TNF therapy) and the presence of infection. The time from commencement of each therapy to onset of infection was recorded. Infections were classified as either major or minor. Major infections were defined as those considered to be systemic, those that required hospitalization and those that directly resulted in the death of the patient. Disease activity was calculated using the HarveyBradshaw Index.

Age groups were compared using chi-square and Wilcoxon Rank Sum statistics. Incidence rate ratios (IRR) of infections in the age groups were calculated. Within age groups, IRRs were calculated for the various medication regimens. Kaplan-Meier survival curves were overlaid to visually compare time to infection. All analyses were completed using SAS 9.3.

\section{$\underline{\text { Results }}$}

Two hundred ninety-two patients were analyzed: 146 were aged 65 or older and 146 were aged less than 65 . In the group aged 65 and older, $55 \%$ were female, $45 \%$ had CD, $45 \%$ had UC and $10 \%$ had IC. The mean age was $73.0(\mathrm{SD}=6.5)$ years, with a range from 65 to 95 years. The mean disease activity, as calculated by the Harvey-Bradshaw equation, was $4.1(\mathrm{SD}=3.0)$. In the group aged less than $65,55 \%$ were female, $47 \%$ had CD, $40 \%$ had UC and $13 \%$ had IC. The mean age was $40.9(\mathrm{SD}=11.6)$ years, with a range from 20 to 64 years. The mean disease activity was $3.9(\mathrm{SD}=2.7)$.

In the younger group, $54(37 \%)$ patients had an infectious complication. Twenty-one of these patients experienced a major infection. In the elderly group, 67 (46\%) patients had an infectious complication. Twenty-seven of these patients experienced a major infection. Of the 292 total patients, 23 patients experienced 2 or more infections. Fourteen of these patients were in the elderly group. No patients died of infectious complications during the time of this study.

A variety of infectious complications were observed during the time of the study (Table 1). One hundred forty-two separate infections occurred in 121 individual patients. There were 23 head and neck infections: 7 cases of sinusitis, 5 cases of oral candidiasis, 4 abscesses, 3 cases of streptococcal pharyngitis, 2 cases of otitis media, 1 case of
Table 1. Types of infections.

\begin{tabular}{|c|c|c|}
\hline Organ system & Infection & Number \\
\hline \multirow[t]{7}{*}{ HEENT $(n=23)$} & Sinusitis & 7 \\
\hline & Thrush & 5 \\
\hline & Head \& Neck abscess & 4 \\
\hline & Strep pharyngitis & 3 \\
\hline & Otitis media & 2 \\
\hline & Otitis externa & 1 \\
\hline & Conjunctivitis & 1 \\
\hline \multirow[t]{2}{*}{ Neurological $(\mathrm{n}=2)$} & Brain abscess & 1 \\
\hline & Meningitis (listeria) & 1 \\
\hline \multirow[t]{25}{*}{ Skin $(n=30)$} & Cellulitis & 16 \\
\hline & Candidiasis & 8 \\
\hline & Onychomycosis & 3 \\
\hline & Wound infection & 2 \\
\hline & Lower extremity ulcer & 1 \\
\hline & URI & 11 \\
\hline & Pneumonia & 10 \\
\hline & Bronchitis & 2 \\
\hline & Tracheitis & 1 \\
\hline & Pneumocystis jiroveci & 1 \\
\hline & MAI & 1 \\
\hline & Perirectal/anal abscess & 13 \\
\hline & C. Diff & 7 \\
\hline & Abdominal abscess & 3 \\
\hline & Diverticulitis & 2 \\
\hline & CMV colitis & 2 \\
\hline & Liver abscess & 1 \\
\hline & Candidal esophagitis & 1 \\
\hline & Necrotizing cholecytitis & 1 \\
\hline & UTI & 20 \\
\hline & Vaginal candidiasis & 3 \\
\hline & Prostatitis & 2 \\
\hline & Tuboovarian abscess & 1 \\
\hline & Bacteremia & 4 \\
\hline & Entaemoba histolytica & 1 \\
\hline
\end{tabular}

otitis externa and 1 case of conjunctivitis. There was 1 case of listeria meningitis and 1 brain abscess. There were 30 skin infections: 16 cases of cellulitis, 8 cases of candidiasis, 3 cases of onychomycosis and 3 wound infections. There were 26 pulmonary infections: 11 upper respiratory tract infections, 10 pneumonias, 2 cases of bronchitis, 1 case of tracheitis, 1 case of pneumocystis jiroveci and 1 case of mycobacterium avium complex. The patient with pneumocystis jiroveci was not on appropriate prophylaxis. There were 30 gastrointestinal infections: 13 perirectal abscesses, 7 cases of clostridium difficile colitis, 3 intraabdominal abscesses, 2 cases of diverticulitis, 1 case of CMV colitis, 1 hepatic abscess, 1 case of candida esophagitis, and 1 case of necrotizing cholecystitis. There were 26 genitourinary infections: 20 urinary tract infections, 3 cases of candida vaginitis, 2 cases of prostatitis and 1 tuboovarian abscess. There were also 4 cases of bacteremia and 1 case of entamoeba histolytica. The most common infection overall was lower urinary tract infections, followed by cellulitis.

Infectious complication occurrences were compared between the younger and elderly patient groups. The infections per patient years of follow up in the younger and elderly groups were 0.106 (95\% CI $=0.081$, $0.132)$ and $0.170(95 \% \mathrm{CI}=0.134,0.206)$, respectively. The IRR for infection when comparing the older group to the younger group was 1.60 (95\% CI=1.16,2.21).

Infectious complications were also compared between specific 
treatment regimens. The infectious complications per patient years of follow up were calculated for each possible treatment regimen (Figure 1). The infectious complications per patient years of follow up for regimens that included corticosteroids, immunomodulators, or TNF inhibitors were 0.21 (95\% CI=0.140, 0.269), 0.11 (95\% CI $=0.076$, $0.146)$, and 0.14 (95\% $\mathrm{CI}=0.094,0.183)$, respectively. The infectious complications per patient years of follow up in patients on any drug therapy and no drug therapy were $0.12(95 \% \mathrm{CI}=0.096,0.150)$ and 0.15 (95\% CI $=0.117,0.187)$, respectively. The infectious complications per patient years of follow up for all patients in the study, whether or not they were on drug therapy, was $0.14(95 \% \mathrm{CI}=0.114,0.157)$. The infectious complications per patient years of follow up for immunomodulators and TNF inhibitors without concomitant corticosteroids were 0.08 (95\% CI $=0.050,0.116)$ and $0.09(95 \% \mathrm{CI}=0.054,0.136)$, respectively (Figure 2). The infectious complications per patient years of follow up for immunomodulators and TNF inhibitors with concomitant corticosteroids were $0.26(95 \% \mathrm{CI}=0.126,0.402)$ and $0.35(95 \%$ $\mathrm{CI}=0.178,0.519)$, respectively.

The IRRs for infectious complications in elderly patients on various therapy regimens were calculated (Table 2). The IRRs for TNF inhibitors, immunomodulators, and corticosteroids when compared to all elderly patients, whether or not they were on drug therapy, were 0.85 $(95 \% \mathrm{CI}=0.47,1.54), 0.78(95 \% \mathrm{CI}=0.44,1.39)$, and $1.84(95 \% \mathrm{CI}=1.16$,

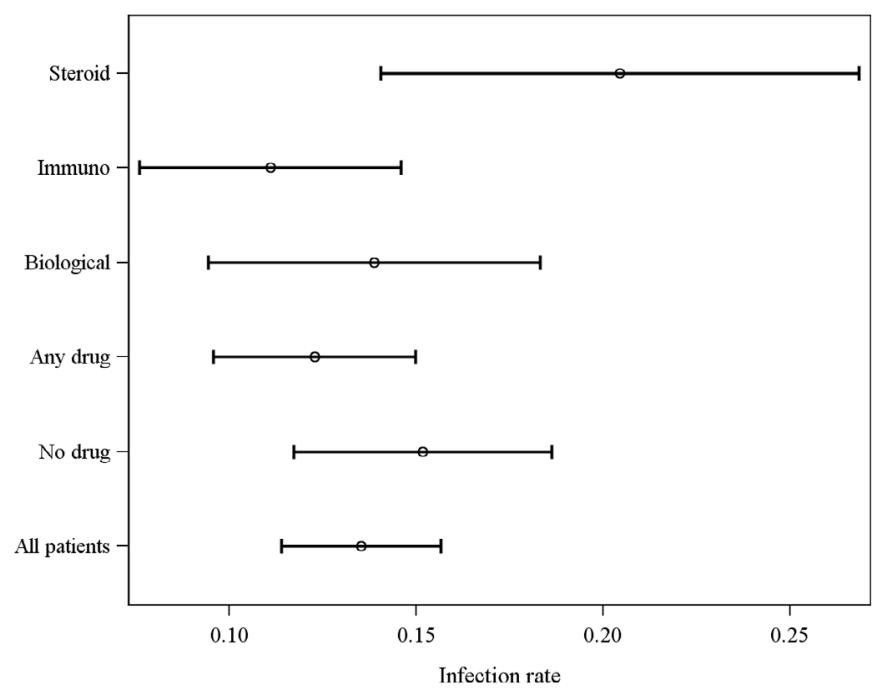

Figure 1. Infectious Complications Per Person Years of Follow up.

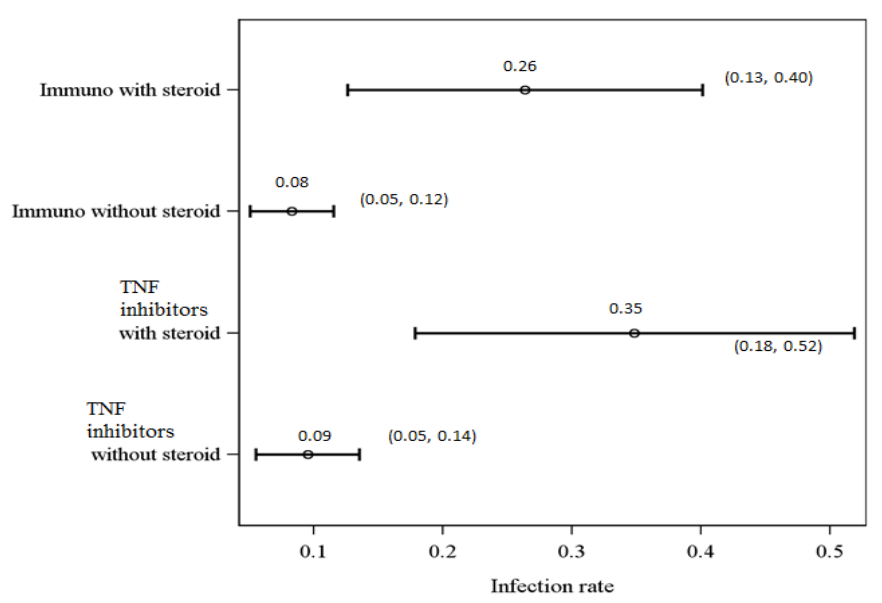

Figure 2. Infectious complication rates with addition of steroids.

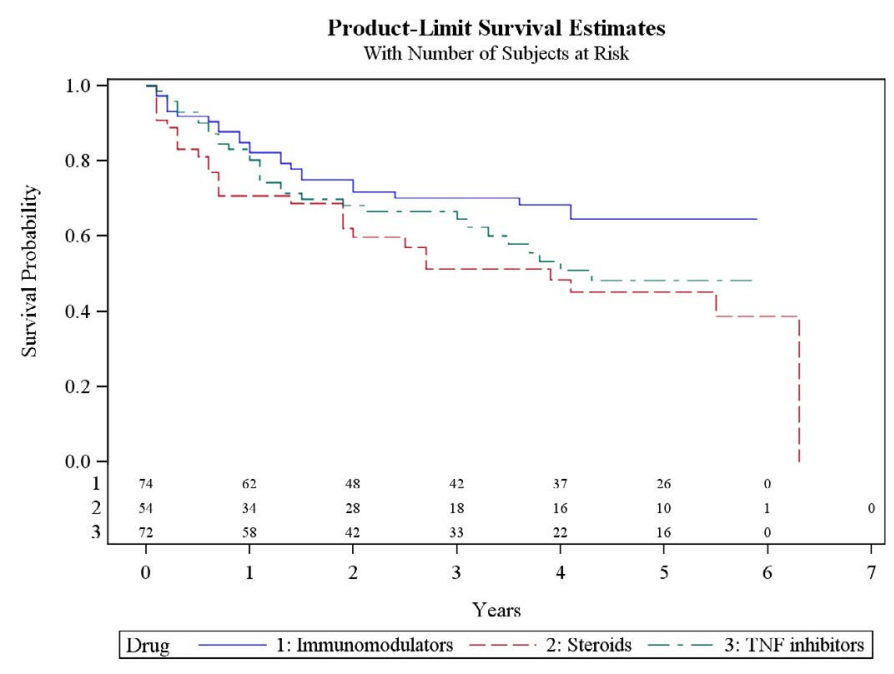

Figure 3. Time from commencement of drug therapy to first infectious complication.

2.91), respectively. The IRRs for TNF inhibitors, immunomodulators, and corticosteroids, when compared to all elderly patients that were on drug therapy, were $0.89(95 \% \mathrm{CI}=0.45,1.74), 0.79(95 \% \mathrm{CI}=0.40,1.53)$, and $3.28(95 \% \mathrm{CI}=1.62,6.64)$, respectively. The IRRs for elderly patients on TNF inhibitors and immunomodulators with the concomitant use of corticosteroids, compared to either drug alone, were 10.64 (95\% CI= $1.95,58.10)$ and $10.58(95 \% \mathrm{CI}=1.27,87.91)$, respectively.

The time to infectious complication from the commencement of each drug was analyzed (Figure 3). The time to infectious complications was shortest in patients on drug regimens that included corticosteroids, followed by those that included TNF inhibitors, and longest in those that included immunomodulators.

\section{Discussion}

This is the first study analyzing infectious complications, specifically in elderly IBD patients while taking the effect of corticosteroids, immunomodulators, and TNF inhibitors into consideration. Elderly age is an independent predictor associated with an increased risk of infectious complication in IBD patients. When compared to their younger IBD counterparts, elderly age increased the risk of infectious complications over 1.5 times. It has been established that elderly IBD patients tend to have worse outcomes when compared with younger IBD patients [15]. Elderly IBD patients on medical therapy have increased mortality [16]. Corticosteroids, immunomodulators, and TNF inhibitors are standard therapies for IBD, as current practice guidelines have reinforced [17]. However, these drugs have not been extensively studied in the elderly IBD population. Many studies have set upper limits on the age of patients and have thus excluded this population from formal analysis. Cottone, et al analyzed the infectious complications of elderly IBD patients on TNF inhibitors [7], but did not take corticosteroids into account. Corticosteroids greatly increase the risk of infectious complications in elderly patients. TNF inhibitors and immunomodulators alone do not significantly increase the risk of infectious complications. However, when corticosteroids are used concomitantly with TNF inhibitors or immunomodulators, the risk of infectious complications appears to increase significantly. Because of the small number of elderly patients on therapy that includes corticosteroids with TNF inhibitors or immunomodulators, definitive conclusions cannot be drawn about the infectious complication risk in this population in our study. The use of immunosuppressants decreases 
Table 2. IRRs of Elderly Patients on Therapy.

\begin{tabular}{|c|c|c|c|c|c|c|c|c|}
\hline \multirow[b]{2}{*}{ Drug type } & \multicolumn{3}{|c|}{ On drug } & \multicolumn{5}{|c|}{ Off drug } \\
\hline & Incidents N & Follow-up years & Incidence rate & Incidents N & Follow-up years & Incidence rate & IRR (On drug/Off drug) & $95 \%$ confidence interval \\
\hline TNFI vs. all patients & 13 & 87.2 & 0.149 & 73 & 418.1 & 0.175 & 0.85 & $0.47,1.54$ \\
\hline IMM vs. all patients & 14 & 100.5 & 0.139 & 72 & 404.8 & 0.178 & 0.78 & $0.44,1.39$ \\
\hline CS vs. all patients & 26 & 96.4 & 0.270 & 60 & 408.9 & 0.147 & $\underline{1.84}$ & $1.16,2.91$ \\
\hline TNFI vs. all meds & 13 & 87.2 & 0.149 & 24 & 143 & 0.168 & 0.89 & $0.45,1.74$ \\
\hline IMM vs. all meds & 14 & 100.5 & 0.139 & 23 & 129.7 & 0.177 & 0.79 & $0.40,1.53$ \\
\hline CS vs. all meds & 26 & 96.4 & 0.270 & 11 & 133.8 & 0.082 & $\underline{3.28}$ & $1.62,6.64$ \\
\hline TNFI+CS vs. TNFI alone & 4 & 8.1 & 0.494 & 2 & 43.1 & 0.046 & $\underline{10.64}$ & $1.95,58.10$ \\
\hline IMM+CS vs. IMM alone & 1 & 1 & 1.000 & 6 & 63.5 & 0.094 & $\underline{10.58}$ & $1.27,87.91$ \\
\hline
\end{tabular}

the time to infectious complications in IBD patients. Corticosteroids had the shortest time from commencement of therapy to the diagnosis of an infection.

There are potential limitations to this study. A substantillay larger population size (likely requiring a multicenter study) would be required to draw definitive conclusions about the infectious complication risk on various combination medication regimens. Only medication use and infections diagnosed during the time of the study were considered. Another potential limitation is the dose and duration of medications were not formally evaluated. In the treatment of other diseases, corticosteroid dose and duration influenced the risk of infectious complications [18]. This finding has not translated to IBD patients when analyzed in most other studies [10,11].

This study suggests that elderly age is an independent risk factor for infectious complications in IBD patients. Some medications, namely immunosuppressants, used to treat IBD may also increase the risk of infectious complications in this patient population. Corticosteroids greatly increase this risk, especially when used concomitantly with immunomodulators and TNF inhibitors. These findings reinforce the importance that risks and benefits be carefully assessed prior to considering use of corticosteroids in all IBD patients, particularly the elderly.

\section{References}

1. Kappelman MD, Moore KR, Allen JK, Cook SF (2013) Recent trends in the prevalence of Crohn's disease and ulcerative colitis in a commercially insured US population. Dig Dis Sci 58: 519-525. [Crossref]

2. Kappelman MD, Rifas-Shiman SL, Kleinman K, Ollendorf D, Bousvaros A, et al. (2007) The prevalence and geographic distribution of Crohn's disease and ulcerative colitis in the United States. Clin Gastroenterol Hepatol 5: 1424-1429. [Crossref]

3. Hämmerlein A, Derendorf H, Lowenthal DT (1998) Pharmacokinetic and pharmacodynamic changes in the elderly. Clinical implications. Clin Pharmacokinet 35: 49-64. [Crossref]

4. Yang YX, Lichtenstein GR (2002) Corticosteroids in Crohn's disease. Am J Gastroenterol 97: 803-823. [Crossref]

5. Sandborn WJ (1996) A review of immune modifier therapy for inflammatory bowel disease: azathioprine, 6-mercaptopurine, cyclosporine, and methotrexate. $\mathrm{Am} \mathrm{J}$ Gastroenterol 91: 423-433. [Crossref]

6. Hochberg MC, Lebwohl MG, Plevy SE, Hobbs KF, Yocum DE (2005) The benefit/risk profile of TNF-blocking agents: findings of a consensus panel. Semin Arthritis Rheum 34: 819-836. [Crossref]

7. Stuck AE, Minder CE, Frey FJ (1989) Risk of infectious complications in patients taking glucocorticosteroids. Rev Infect Dis 11: 954-963. [Crossref]

8. Sandborn WJ (2010) State-of-the-art: Immunosuppression and biologic therapy. Dig Dis 28: 536-542. [Crossref]

9. Gordon R, Mays R, Doan H, Lapolla W, Tyring SK (2012) Biologic therapy and risk of infection. Skin Therapy Lett 17: 1-4. [Crossref]

10. Lichtenstein GR, Feagan BG, Cohen RD, Salzberg BA, Diamond RH, et al. (2006)
Serious infections and mortality in association with therapies for Crohn's disease: TREAT registry. Clin Gastroenterol Hepatol 4: 621-630. [Crossref]

11. Lichtenstein GR, Feagan BG, Cohen RD, Salzberg BA, Diamond RH, et al. (2012) Serious infection and mortality in patients with Crohn's disease: more than 5 years of follow-up in the TREATâ,, $\notin$ registry. Am J Gastroenterol 107: 1409-1422. [Crossref]

12. Aberra FN, Lewis JD, Hass D, Rombeau JL, Osborne B, et al. (2003) Corticosteroids and immunomodulators: postoperative infectious complication risk in inflammatory bowel disease patients. Gastroenterology 125: 320-327. [Crossref]

13. Cottone M, Kohn A, Daperno M, Armuzzi A, Guidi L, et al. (2011) Advanced age is an independent risk factor for severe infections and mortality in patients given anti-tumor necrosis factor therapy for inflammatory bowel disease. Clin Gastroenterol Hepatol 9: 30-35. [Crossref]

14. Charpentier C, Salleron J, Savoye G, Fumery M, Merle V, et al. (2014) Natural history of elderly-onset inflammatory bowel disease: a population-based cohort study. Gut 63 : 423-432. [Crossref]

15. Stallmach A, Hagel S, Gharbi A, Settmacher U, Hartmann M, et al. (2011) Medical and surgical therapy of inflammatory bowel disease in the elderly - prospects and complications. J Crohns Colitis 5: 177-188. [Crossref]

16. Ananthakrishnan AN, McGinley EL, Binion DG (2009) Inflammatory bowel disease in the elderly is associated with worse outcomes: a national study of hospitalizations. Inflamm Bowel Dis 15: 182-189. [Crossref]

17. Clark M, Colombel JF, Feagan BC, Fedorak RN, Hanauer SB, et al. (2007) American gastroenterological association consensus development conference on the use of biologics in the treatment of inflammatory bowel disease, June 21-23, 2006. Gastroenterology 133: 312-339. [Crossref]

18. Klein NC, Go CH, Cunha BA (2001) Infections associated with steroid use. Infect Dis Clin North Am 15: 423-432. [Crossref]

Copyright: (C2016 Tofani C. This is an open-access article distributed under the terms of the Creative Commons Attribution License, which permits unrestricted use, distribution, and reproduction in any medium, provided the original author and source are credited. 